\title{
CORRIGENDA
}

\section{Antenatal prediction of postpartum depression with blood DNA methylation biomarkers}

J Guintivano, M Arad, TD Gould, JL Payne and ZA Kaminsky

Molecular Psychiatry (2014) 19, 633; doi:10.1038/mp.2013.149; published online 22 October 2013

Correction to: Molecular Psychiatry advance online publication, 21 May 2013; doi:10.1038/mp.2013.62

Following the online publication of this paper, the authors noticed that Supplementary Table 2 contains incorrect oligonucleotide primer sequences. The corrected table accompanies this corrigendum online.

Supplementary Information accompanies the paper on the Molecular Psychiatry website (http://www.nature.com/mp)

\section{Role of brain transmigrating neutrophils in depression-like behavior during systemic infection}

A Aguliar-Valles, J Kim, S Jung, B Woodside and GN Luheshi

Molecular Psychiatry (2014) 19, 633; doi:10.1038/mp.2013.154; published online 5 November 2013

Correction to: Molecular Psychiatry, advance online publication, 15 October 2013; doi:10.1038/mp.2013.137
After the above article was published, the authors noted that the first author was listed incorrectly. The author's correct name is A Aguilar-Valles. 